\title{
Analysis on Tourism Environmental Pollution and Tourism Economy-Ecological Environmental Coordination Degree: A Case Study from China
}

\author{
Zhong Wei Wang† and Huan Le Han \\ Yellow River Conservancy Technical Institute, Kaifeng 475004, Henan, China \\ $\dagger$ Corresponding author: Zhongwei WANG, Email: 94381557@qq.com
}

\section{Nat. Env. \& Poll. Tech.}

Website: www.neptjournal.com

Received: 15-06-2021

Revised: 21-07-2021

Accepted: 01-08-2021

Key Words:

Tourism

Environmental pollution

Coordination degree

Shandong province

\begin{abstract}
The rapid development of the tourism industry, range expansion, and intensity growth of human tourism activity have all caused intensifying ecological environmental problems while bringing considerable economic benefits. The ecological environment is currently faced with huge pressures due to the unreasonable tourism resource development, annual decrease in bearing capacity of the tourism environment, and excessive tourism consumption. To coordinate the relationship between tourism economic growth and ecological environment, this work carried out a case study based on Shandong Province, China. First, four aspects of environmental pollution caused by tourism were analyzed. Second, the tourism economy-ecological environmental coordination degree model and index system were established and then the coordination degree in Shandong Province from 2002 to 2019 was estimated. Results show that the tourism industry causes the following typical pollutions: water, soil, air, and noise. The coupling coordination degree (D) between the tourism economy and ecological environment in Shandong Province increased from 0.100 in 2002 to 0.995 in 2019, indicating the transition from serious imbalance to a high-quality coordination state. It is suggested to further promote tourism economy-ecological environment coordinated development by innovating the tourism-economy coupling development mode, creating the entire tourism industrial chain, increasing efforts in tourism reform, and exploring and implementing ecological compensation policies. Research conclusions have important significances to further study and explore the development status between the tourism industry and ecological environment and realize the coordinated development between the tourism industry and regional economy.
\end{abstract}

\section{INTRODUCTION}

The tourism industry has globally become the largest economic industry. With the rapid development of the global tourism economy and China's national economy, tourism has become one of the pillar industries to China's economic development. Recently, China has published many relevant policies to deepen reform in the opening of the tourism industry, accelerate its upgrading, optimize its structure, and support its development, facilitating its advancement into a strategic pillar industry of China's economic development and modernized service industry. However, with the development of the tourism industry, environmental problems caused by tourism economic activities have been a huge concern. Owing to unreasonable development and overload operation, tourism resource and ecological environment suffer excessive impacts, thus causing a series of environmental problems, including tourism-induced environmental pollutions (e.g., water, soil, noise, and waste pollutions), landscape hazards, and decreased biodiversity, among others.

Shandong Province, China is a province with high economic development and tourism development in Eastern
China. It possesses a huge tourism industry scale and accepts numerous foreign and Chinese tourists. In Fig. 1, the foreign exchange earnings from tourism (million dollars) and reception of international tourists (million) increased each year from 2009 to 2019, with annual average growths of distribution at $9.33 \%$ and $3.03 \%$, respectively. With the continuous growth of tourism income and tourists, the tourism industry has brought relatively serious environmental pollution. Given that the tourism industry in Shandong Province belongs to a typical resource and environment-dependent industry, the tourism activity scale in some regions disagrees with local environmental capacity. Hence, tourism resources are hazards, which are against the sustainable development in tourism regions in Shandong Province. Some tourism developers are only concerned with immediate interests and ignore environmental problems in the development process. Moreover, public consciousness of green tourism is weak, thus continuously increasing the carbon emissions of the entire tourism industry. The rapid development of the tourism industry not only promotes regional economic growth but also brings with it some environmental problems. The ecological environment of Shandong Province is vulnerable, and 


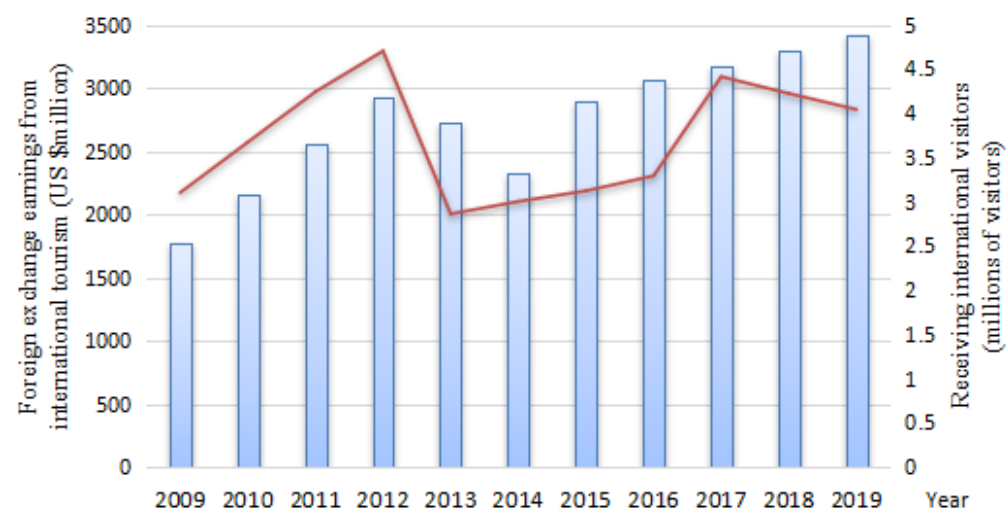

Fig. 1: Foreign exchange earnings from tourism and reception of international tourists during 2009-2019.

the effects of tourism activities on the ecological environment will be extremely obvious. Therefore, exploring the coupling relationship and mechanism between the tourism economy and ecological environment in Shandong Province, estimating the coupling development level of the two systems, and proposing suggestions for coordinated development are the necessary steps.

\section{PAST STUDIES}

Tourism-induced pollution hazards and the coordinated development between the tourism economy and ecological environment have become a hotspot in the sustainable development of the tourism industry. Since 1980, Chinese and foreign researchers have begun reporting many studies on environmental pollution and ecological safety problems in forest tourist attractions. In the aspect of tourism-induced pollution hazards: Greiner et al. studied the influences of environmental pollution on the optimization development of the tourism industry. Research results demonstrated that environmental pollution presents periodic effects on the tourism industry, and tourism activities decreased when heavy environmental pollution occurs (Greiner et al. 2001). Kocasoy et al. (2008) believed that the summer resort in the coastal regions discharges wastewater into the sea without any processing, thus worsening the water area in coastal regions. A case study based on two beaches in Turkey was carried out. Results showed that the direct discharge of wastewater in scenic areas into the marine environment without any processing led to serious seawater pollution. Jang et al. studied that abundant marine wastes were washed up to the beaches of Jiejie Island in Korea and influence the tourism industry of the island. This pollution event led the tourism industry to lose approximately 29-37 million dollars of revenue (Jang et al. 2014). Zhang et al. discussed the influences of the international tourism industry on China's energy consumption by replacing environmental pollution with $\mathrm{CO}_{2}$ emission in the multi-variable framework based on the regional panel data from 1995 to 2011. Results demonstrated that the long-term effects of the tourism industry on $\mathrm{CO}_{2}$ emission are a causal relationship (Zhang et al. 2016). Wilson et al. analyzed the influences of the tourism industry on the south of the Great Barrier Reef, and it was found to contribute to the litter level in the local areas. He found several tourism-sourced goods at places near the amenities, which led to waste pollution (Wilson et al. 2017). Ahmad et al. deemed that the rapid economic development in China and industrial transfer from East China to West China caused serious negative effects. According to research results, the tourism industry has negative impacts on the environments in Ningxia, Qinghai, Gansu, and Shanxi (Ahmad et al. 2018). Zeng et al. believed that unreasonable tourism development and huge energy consumption brought serious challenges to the urban environment in China. According to the results, the direct effects of tourism industrial development on the PM2.5 concentration presented an inverted U-shaped relation (Zeng et al. 2020). In the coordinated development between tourism economy and ecological environment, Huang et al. investigated the interactions between the tourism industry and mining industry in terms of economic contributions and spatial patterns in the biodiversity hotspot regions of Yunnan Province. It found that the tourism industry concentrates in underdeveloped regions, and it is an important industry to realize local economic development (Huang et al. 2011). Yuan et al. constructed the evaluation index system of the regional environment-tourism-economy coordinated development by using the pressure-state-response (PSR) model. Moreover, an empirical analysis on the coordinated development status between the environment and tourism economic system in western Hunan from 2001 to 2012 was carried out. Results showed that although the environment-tourism econom- 
ic system in western Hunan has not reached a relatively high-efficiency index, the coupling degree among subsystems was not high (Yuan et al. 2014). Zheng et al. evaluated the comprehensive indexes and coordinated relationship between tourism economy and traffic system in 9 cities of the Pearl River Delta (PRD) by using the comprehensive evaluation model and coupling coordination model. Results showed that the tourism economy and traffic transportation in 9 cities of the PRD tended to form a coordinated development gradually, and the tourism economy in the central area of PRD was quicker than those in other regions (Zheng et al. 2016). Luo et al. carried out an expanded study on low-carbon tourist attractions and low-carbon tourism in Jiuzhaigou Valley by using the decarbonated development in the system of tourist attractions (DDSTA) index reference set and application set based on management entropy (Luo et al. 2017). Based on the sensitivity recovery pressure (SRP) framework, Kan et al. established the vulnerability evaluation system in the plateau tourist areas and provided a model that can be promoted to ecological vulnerability evaluation during tourism planning and development in high mountain regions (Kan et al. 2018). Lai et al. established the comprehensive evaluation index system of ecological environment, economy, and tourism industry, and calculated the coupling degree and coordination degree of 3 subsystems in 31 provinces in China from 2003 to 2017. Results showed that the mean coupling degree and mean coordination degree experienced a fluctuating upward trend from 2003-2017 (Lai et al. 2020). Yang et al. defined the restoration of urban tourism enterprises from the perspectives of social, economic, and ecological environments and constructed an evaluation index system of restoration power of urban tourism enterprises (Yang et al. 2021). Li et al. proposed the comprehensive evaluation index system of tourism development levels from the aspects of market scale, tourism economy, tourism resource, and tourism service. A coupling coordination degree model (CCDM) of tourism industry, urbanization, and ecological environment were built with panel data of Chongqing City from 2000 to 2017. As time goes on, the coupling coordination degree of the three systems increased progressively (Li et al. 2021). Burger et al. believed that the tourism industry can strengthen land protection on the landscape scale, thus protecting habitat and biodiversity (Burger et al. 2000). According to the existing literature review, Chinese and foreign scholars have achieved some research fruits on the relationship between the tourism industry and the regional economy. In China, studies focus on the transverse comparison between the tourism industry and regional economic development, but studies on longitudinal comparison and evolutional analysis have to be promoted. Moreover, the scientificity of the built comprehensive evaluation index system requires further discussion.
Therefore, this study carried out a quantitative study on the coupling relation between tourism economy and ecological environment in Shandong Province, China, aiming to enrich the empirical range for studying tourism economy-ecological environment coordinated development. This study can provide some theoretical references to examine tourism in the study area and offer some policy suggestions to tourism economy-ecological environment coordinated development in Shandong Province.

\section{TOURISM-INDUCED ENVIRONMENTAL POLLUTION HAZARDS}

\section{Water Pollution}

Owing to tourism activities and sewage from the production and operation in the scenic areas, organic contents, mainly total nitrogen (TN) and total phosphorus (TP), increase significantly. In addition, substantial chemicals, such as cleansers and disinfectant fluid, are discharged into water bodies in scenic areas due to the affiliated industrial pollution discharge of tourism. Several pollutants continuously enter the water bodies of the scenic areas, thus causing water eutrophication and significantly increasing the microbial flora and suspended particles as well as reproductions of algae and fecal coliform. Boats and transport machines floating on the waters in the scenic areas are typically powered by diesel. The tail gas may cause water pollution, and poor maintenance of boat structures may cause diesel leakage which causes serious pollution to water bodies in scenic areas.

\section{Soil Pollution}

In tourism activities, the land surface surrounding arteries becomes bare due to the continuous stamping by tourists. Many problems result due to extensive loss of soil water, such as soil hardening, porosity reduction, permeability reduction, and increase in soil bulk density. The huge loss of soil water and increasing hardening degree weaken the adsorption of microelements in the soil surface layer and thus decrease organic contents and enzyme activity in the soil. Influenced by erosion of tourism lines, some regions have undergone serious soil degeneration. Construction of tourism attractions, tourist stamping, and unqualified garbage processing technology will also cause evident changes in the soil $\mathrm{pH}$. Moreover, chemical reagents used by agricultural production near the tourism attractions, household wastes of tourists, and traffic tools can all increase the heavy metal contents in soils.

\section{Air Pollution}

The use of traffic tools, catering, hotel, and processing in 
tourism attractions as well as harmful gases and substances represented by $\mathrm{CO}, \mathrm{SO}_{2}$, and $\mathrm{Pb}$ produced during the use of coal fuels in nearby factories can all worsen the air environment condition in the tourism attractions. Among them, $\mathrm{SO}_{2}$ pollution which causes acidification of soils is the most serious. In the operation process of tourism attractions, garbage piling and fermentation caused by the poor disposal technology release considerable amounts of harmful gases, including methane and xylene. Suspended particles, such as flying dust and powder produced in the construction and development processes of tourist attractions as well as the smoke produced by relevant activities of affiliated industries in the scene, can all cause air pollution in the tourism scene.

\section{Noise Pollution}

The service process of tourism attractions produces excessive noise given that facilities and equipment such as roller coasters, boats, air conditioners, and machine rooms for tourism activities, consume substantial amounts of energy sources. Moreover, the power system and whistle of traffic tools, such as tour buses, sightseeing buses, self-driving cars, and trucks in the tourism attractions, from excessive noise. Tourist centers in the tourism attractions accumulate excessive noise due to the pronounced concentration or uproars of tourists. Different noises not only influence the tourism experiences of tourists but also affect the survival environment of animals and plants in the tourism attractions. The peak season of tourism activities in tourism attractions coincides with the season for animal and plant growth and development. Misconduct in the sightseeing process of tourists also brings notable impacts on the reproduction, migration, and other living habits of animals and may even cause their diseases and deaths.

\section{MODEL INTRODUCTION AND INDEX SYSTEM}

\section{Coordination Degree Model}

Estimation of coordination degree: Considering that two subsystems $S_{j}, j \in[1, k]$ of tourism economy and ecological environment are studied, the order parameter variables in the development process of these two subsystems are $e_{j}=\left(e_{j 1}, e_{j 2}, \cdots, e_{j n}\right)$, where $n \geq 1, \beta_{j i} \leq e_{j i} \leq \alpha_{j i}, i \in[1, n]$. Without loss of generality, we suppose that the order degree of systems is positively related to the value of $e_{j 1}, e_{j 2}, \cdots, e_{j l}$ and is negatively related to the value of $e_{j l+1}, e_{j l+2}, \cdots, e_{j n}$. The tourism economy and ecological environmental system of Shandong Province include the Shandong tourism economic subsystem and Shandong ecological environmental subsystem. The order parameters in the development process of the tourism economic subsystem are $e_{1}=\left(e_{11}, e_{12}, \cdots, e_{1 n}\right)$. According to the concept of order degree of the system, we defined the order degree of order parameter component $e_{1 j}$ of the tourism economic subsystem as:

$$
u_{1}\left(e_{1 i}\right)=\left\{\begin{array}{c}
\frac{e_{1 i}-\beta_{1 i}}{\alpha_{1 i}-\beta_{1 i}}(i=1,2, \cdots l) \\
\frac{\alpha_{1 i}-e_{1 i}}{\alpha_{1 i}-\beta_{1 i}}(i=l+1, l+2, \cdots n)
\end{array}\right.
$$

Where $\alpha_{1 i}$ and $\beta_{1 i}$ are the maximum and minimum of index $i$ of the tourism economic subsystem. According to this definition, we can know that $u_{1}\left(e_{1 i}\right) \in[0,1]$ after normalization. The higher value indicates the greater contribution of $e_{1 i}$ to the order of tourism economic subsystem. In general, the total contributions of order parameters to the order degree of the tourism economic subsystem can be realized through the integrated approach of $u_{1}\left(e_{1 i}\right)$. First, the weights of different indexes have to be determined. To increase the scientificity of index weight, the classical CRITIC (CRiteria Importance Through Intercriteria Correlation) objective weighting method was applied. Diakoulaki D et al. proposed the CRITIC method (Diakoulaki et al. 1995):

$$
\xi_{j}=\sigma_{j} \times \sum_{j=1}^{n}\left(1-r_{i j}\right)(j=1,2,3, \cdots, n)
$$

where $\xi_{j}$ is the influence degree of the evaluation index $j$ on the system. $\sigma_{j}$ is the standard deviation of the evaluation index $j . r_{i j}$ is the correlation coefficient between evaluation indexes $i$ and $j$. The higher numerical value of $\xi_{j}$ indicates the greater influence degree of the evaluation index $j$ on the system. Hence, the calculation formula of objective weight $\omega_{j}$ of the evaluation index $j$ is

$$
\omega_{j}=\frac{\xi_{j}}{\sum_{j=1}^{n} \xi_{j}}(j=1,2,3, \cdots, n)
$$

Where $\omega_{j}$ denotes the weight of different indexes. Apparently, the order degree of tourism economic subsystem is $u_{1}\left(e_{1}\right) \in[0,1]$. The higher value of $u_{1}\left(e_{1}\right)$ indicates the greater contributions of $e_{1}$ to the order degree of the system. Thus, the order degree of the tourism economic subsystem is higher; otherwise, it is lower. Suppose the order parameter in the development process of ecological environmental subsystem is $e_{2}=\left(e_{21}, e_{22}, \cdots, e_{2 i}\right)$. Similarly, the order degree of the parameter component of ecological environmental subsystem $e_{2 i}$ can be obtained according to the calculation method of the order degree of tourism economic subsystem, which is $u_{2}\left(e_{2 i}\right): u_{2}\left(e_{2 i}\right) \in[0,1]$. Suppose the order degree of tourism economic subsystem is $u_{1}^{0}\left(e_{1}\right)$ and the order degree of ecological environmental subsystem is $u_{1}^{1}\left(e_{1}\right)$ at the initial moment $t_{0}$. When the system evolves to $t_{1}$, the order degree of tourism economic subsystem is $u_{1}^{1}\left(e_{1}\right)$, and the order 
degree of ecological environmental subsystem is $u_{2}^{1}\left(e_{2}\right)$. If $u_{1}^{1}\left(e_{1}\right) \geq u_{1}^{0}\left(e_{1}\right), u_{2}^{1}\left(e_{2}\right) \geq u_{2}^{0}\left(e_{2}\right)$ are simultaneously proven, the tourism economy and ecological environment combined system is coordinately developed, and the coordination degree model is shown as follows.

$$
c_{2}=\left\{\left(u_{1} \times u_{2}\right) /\left[\left(u_{1}+u_{2}\right) \times\left(u_{1}+u_{2}\right)\right]\right\}^{1 / 2}
$$

Where $c_{2}$ represents the coupling degree of the combined system. $u_{1}$ and $u_{2}$ are order degrees of tourism economic subsystem and ecological environmental subsystem, respectively.

$$
D=\sqrt{C \times T}, T=\alpha u_{1}+\beta u_{2}
$$

where $D$ is the coupling coordination degree of the combined system. T refers to the comprehensive evaluation index of tourism economy and ecological environment. $\alpha$ and $\beta$ are coefficients for determining, which are generally determined as 0.5 and 0.5 , respectively. Some differences exist in the mutual promotion degrees of subsystems in the combined system.

Grading of coordination degree: The coordination degree model involves the calculation of 3 indexes, namely, the coupling degree (C), coordination index $(\mathrm{T})$, and coupling coordination (D). Finally, the coupling coordination degrees of different indexes are obtained through D and coordination grading standards. The coupling coordination degree originated from the capacity coupling coefficient model in physics. $\mathrm{C}$ value is calculated. The higher value indicates the greater interaction between the two subsystems. $\mathrm{D}$ value is equal to the product of $\mathrm{C}$ value and $\mathrm{T}$ value, and it ranges between $0-1$. The higher value of $\mathrm{D}$ indicates the higher coordination degree between the two subsystems.

\section{Index System}

The tourism economy and ecological environment coupling evaluation index system shall involve tourism economic and ecological environmental development levels. Based on meeting the design principle of comprehensive, objective, operable, quantitative, and qualitative indexes, Table 1 shows an evaluation index system of tourism economy-ecological environmental coordination degree built preliminarily through a comprehensive analysis of definition connotations, influencing factors, and structural characteristics of tourism economy and ecological environment and by combining frequency analysis of evaluation indexes in existing studies. Additionally, the investigation period is from 2002 to 2019.

\section{EMPIRICAL STUDY}

According to the coupling evaluation model and formula, this study estimated and analyzed the tourism economy and ecological environment coupling development in Shandong Province. Fig. 2 shows the results. As seen from Fig. 2, the comprehensive evaluation level of the tourism economy in Shandong Province generally presents a fast growth trend, and the comprehensive evaluation level of the tourism economy significantly increases from 0.0002 in 2002 to 0.5908 in 2019. In this period, the economic growth rate was relatively high in Shandong Province. Accordingly, the growth rates of tourism income and resident income were in a high-level growing trend. Tourism plans, such as the "13th Five-year" Plan for Tourism Industrial Development of Shandong Province, were approved and implemented successively. These steps can all promote the sound and reasonable development of the tourism industry in Shandong Province. At the same time, Shandong Province was making considerable efforts to promote the integrated development of tourism, facilitating the transformation and upgrading of international tourism and guiding the "tourism + " mode, thus realizing rapid tourism economic growth in the province. From 2002 to 2019 , the comprehensive evaluation level of the ecological

Table 1: Coupling coordination grading standards.

\begin{tabular}{lll}
\hline Interval of D & Coordination grade & Coupling coordination degree \\
\hline$(0.0-0.1)$ & 1 & Extreme imbalance \\
{$[0.1-0.2)$} & 2 & Serious imbalance \\
{$[0.2-0.3)$} & 3 & Moderate imbalance \\
{$[0.3-0.4)$} & 4 & Slight imbalance \\
{$[0.4-0.5)$} & 5 & Endangered imbalance \\
{$[0.5-0.6)$} & 6 & Poor balance \\
{$[0.6-0.7)$} & 7 & Primary balance \\
{$[0.7-0.8)$} & 8 & Moderate balance \\
{$[0.8-0.9)$} & 9 & Good balance \\
{$[0.9-1.0)$} & 10 & High-quality balance \\
\hline
\end{tabular}


environment in Shandong Province highly fluctuated and developed from 0.002 in 2002 to 0.8337 in 2019. Owing to the continuous development of the tourism economy, tourism activities cause a huge ecological environmental pressure, while the comprehensive evaluation level of the ecological environment remains low. Shandong Province successively issued environmental protection regulations in the process of international tourism destinations and comprehensive reform pilot zones of tourism by providing substantial capital funds to environmental protection control. These regulations somewhat promoted the improvement of the ecological environment. Government intensity policies and measures for pollution control and the industrial structure and resource configuration are optimized. Consequently, the comprehensive evaluation level of Shandong Province began to increase again.

As seen from Table 3, the $\mathrm{D}$ value increased from 0.100 in 2002 to 0.995 in 2019, indicating that tourism and ecology in Shandong Province have developed from extreme imbalance in 2002 to high-quality balance in 2019. Recently, China has issued many policy documents that support ecological protection and tourism industry integrated development.
Shandong Province created a unique cultural experience, designed participated experiencing projects, and enriched the tourism product system by strengthening tourism development of cultural sites and relics using high-tech tools. Shandong Province built a group of high-quality resort areas, improved and created the national-level resort areas, and strengthened the dynamic management of provincial resort areas. Guided by building a good traditional cultural tourism base and a red cultural tourism base, the new advantages of cultural tourism in Shandong Province are created. Meanwhile, promoting and implementing the infrastructure construction of poverty alleviation pilots via tourism have begun. In Shandong Province, rural tourism resource points are enriching continuously, and tourism destinations available for tourists are increasing and thus effectively lowering the tourist density in key scenic areas and relieving ecological environmental pressure.

\section{POLICY SUGGESTIONS}

\section{Innovate the Tourism Economic Coupling Development Mode and Relieve Ecological Pressure}

The technology-dependent independent innovative tourism

Table 2: Evaluation index system of tourism economy and ecological environment coordination degree.

\begin{tabular}{ll}
\hline Indexes of tourism economic subsystem (Unit) & Indexes of ecological environmental subsystem (Unit) \\
\hline Number of received inbound tourists staying overnight (people) & Daily handling capacity of urban sewage (10,000 $\mathrm{m}^{3}$ ) \\
Number of days of received inbound tourists staying overnight and days (people/day) & Urban Greenland area (ha.) \\
Average stay of received inbound tourists (days) & Park area (ha.) \\
Number of star-rated hotels (hotels) & Cleaning area (10,000 $\left.\mathrm{m}^{2}\right)$ \\
Labor productivity of total workers (1,000 yuan/people) & Amount of household waste clean-up (10,000 tons) \\
$\begin{array}{l}\text { Per capita original value of fixed assets (1,000 yuan/people) } \\
\text { Revenues from } 100 \text { yuan of fixed assets (yuan) }\end{array}$ & Wash rooms (rooms) \\
Average occupancy rate of rooms (\%) & Per capita green land area in parks ( $\left.\mathrm{m}^{2}\right)$ \\
Total number of travel agencies (agencies) & Harmless handling capacity (tons/day) \\
Total number of tourism colleges (colleges) & Natural reserve area (10,000 ha.) \\
& $\begin{array}{l}\text { Finished investment for industrial pollution control } \\
\text { (100 million Yuan) }\end{array}$ \\
\hline
\end{tabular}

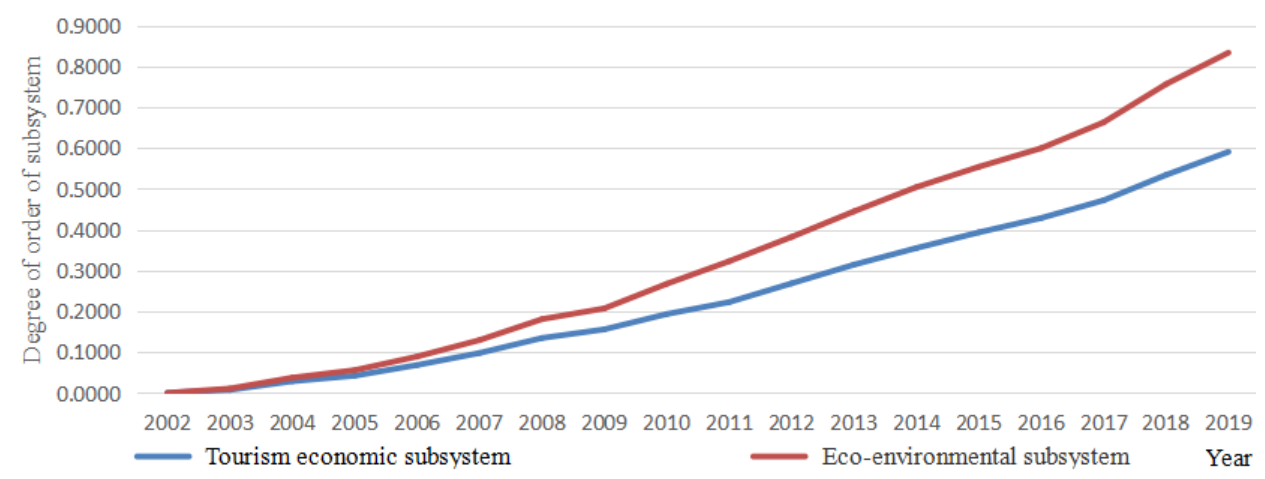

Fig. 2: Order degrees of tourism economic subsystem and ecological environmental subsystems. 
economic coupling development mode is applied in core areas composed of regions with increasing coupling coordination, such as Weihai, Qingdao, and Yantai with highly developed infrastructure and traffic conditions and in the leading position of comprehensive potentials of the tourism industry. These areas shall use tourism industrial development as the bright spot of economic development under a new normal condition. As an important breakthrough that promotes local economic and social development, the tourism industry becomes one of the new dominant kinetic energies driving regional economic development. Finally, the coupling coordination development between the tourism industry and regional economy is realized. Further attention shall be paid to the environmental construction of industrial institutions and fundamental supports. The regulation effect of market mechanism and openness to foreign trade shall be increased. The internal growth potential of the tourism industry is improved by increasing the agglomeration effect and innovation ability. In addition, the market expansion potential of the entire industry is enhanced by guiding and mining market demand potentials. It is suggested to innovate the state of the tourism industry, increase industrial points, deviate industry chain, expand the industrial surface, and construct industrial clusters. New benefit modes are formed by deepening development.

\section{Create the Entire Chain of Tourism Industry and Reduce Resource Waste}

Establishing large-scale tourism groups through merge \& acquisitions, creating a complete industrial chain, and increasing the levels of economic scale and range of the entire tourism industry. Centered at tourism demand, it shall strengthen mutual integration and permeation of the tourism industry and other industries to form an integrated development mode. With respect to integration mode, the tourism industry can be embedded into relevant industries, and these industries are endowed with tourism functions, thus realizing grafting between tourism industrial elements and other industries. The transboundary integration between tourism and various industries is promoted to drive the development of relevant industries. It gives birth to an infinite space for industrial development and facilitates tourism development in the entire region, thus increasing the coupling coordination degree between the tourism industry and regional economy to the coordination rising type. Different cities in Shandong Province shall focus on the construction and perfection of the basic elements of the tourism industry according to practical conditions. They can develop some new tourism products that are independent of tourism resources, such as festival travel and rural tourism, to expand the scope of the tourism industry and provide other practical pathways for tourism industrial development.

Table 3: Calculated results of coupling coordination degree.

\begin{tabular}{|c|c|c|c|c|}
\hline Year & $\mathrm{C}$ value & $\mathrm{T}$ value & $\mathrm{D}$ value & Coupling coordination degree \\
\hline 2002 & 1 & 0.010 & 0.100 & Extreme imbalance \\
\hline 2003 & 1 & 0.022 & 0.150 & Extreme imbalance \\
\hline 2004 & 0.999 & 0.056 & 0.236 & Moderate imbalance \\
\hline 2005 & 1 & 0.078 & 0.279 & Moderate imbalance \\
\hline 2006 & 0.999 & 0.119 & 0.345 & Slight imbalance \\
\hline 2007 & 1 & 0.167 & 0.409 & Endangered imbalance \\
\hline 2008 & 1 & 0.228 & 0.477 & Endangered imbalance \\
\hline 2009 & 1 & 0.261 & 0.511 & Poor balance \\
\hline 2010 & 1 & 0.327 & 0.572 & Poor balance \\
\hline 2011 & 1 & 0.384 & 0.620 & Primary balance \\
\hline 2012 & 1 & 0.457 & 0.676 & Primary balance \\
\hline 2013 & 1 & 0.532 & 0.729 & Moderate balance \\
\hline 2014 & 1 & 0.601 & 0.775 & Moderate balance \\
\hline 2015 & 1 & 0.662 & 0.814 & Good balance \\
\hline 2016 & 1 & 0.718 & 0.847 & Good balance \\
\hline 2017 & 1 & 0.791 & 0.889 & Good balance \\
\hline 2018 & 1 & 0.898 & 0.948 & High-quality balance \\
\hline 2019 & 1 & 0.990 & 0.995 & High-quality balance \\
\hline
\end{tabular}




\section{Increase Tourism Reform Strength and Advocate Green Tourism}

Government at all levels, environmental protection and tourism department, tourism consumers, and relevant tourism enterprises in Shandong Province shall run through the philosophy of low-carbon environment-friendly tourism. Government and environmental protection sectors shall strengthen environmental monitoring and fulfil the environmental protection supervision. The government and tourism department shall make reasonable tourism plans, perform scenic area division and public tourism tool distribution among different scenic spots during the peak season of tourism, and relieve ecological pressure in the scenic spots. Tourism enterprises shall take the initiative in using environment-friendly materials and try to decrease waste-water and waste gas emissions in the production and management process. Tourists shall be aware of carrying bags and cups to reduce garbage production in the scenic areas. They shall try to choose environment-friendly public transport means and protect historical relics, landscape buildings, animals, and plants well during their travels. Scenic spots shall be far away from the pollution discharge enterprises, and the number of parking cars in the scenic areas shall be controlled strictly during the peak season of tourism. It shall advocate green transport means such as bikes, strengthen noise detection in cities, prohibit long-term whistles in scenic spots, and create a relaxed and quiet travelling environment. Scenic spots and mountain resorts shall be equipped with adequate garbage tins and environment-friendly washrooms, and all garbage shall undergo a harmless and timely disposal process.

\section{Strengthen Publicity Education and Explore the Implementation of Ecological Compensation Policy}

Owing to the rapid industrial development in modern times, many enterprises and masses excessively pursue economic benefits while maintaining weak consciousness of ecological environmental protection, thus resulting in forest degeneration and frequent occurrences of natural disasters and different degrees of damages to tourism ecological environment. Environmental protection and publicity require further strengthening. Government at all levels and environmental protection departments of Shandong Province can make publicity and education activities on environmental protection by using the Environmental Day and National Science Popularization Day and spread knowledge about ecological environmental protection via online We-media, radio programs, and TV shows to strengthen public consciousness regarding ecological environmental protection. The tourism department shall strengthen business ability training for environmental protection workers in the scenic spots. It should also encourage tour guides to spread tourism environmental protection knowledge when introducing the landscapes and advocate tourism enterprises to provide green and environment-friendly tourism products and services. Shandong Province shall explore the marketized ecological compensation mode of tourism and standardize the ecological resource market. An ecological compensation fund shall be set up. In particular, attention shall be paid to compensations for tourism ecological managers and local residents and relieving benefit imbalance among construction parties and ecological environmental protection.

\section{CONCLUSIONS}

Environmental problems become increasingly prominent due to behaviors such as the continuous booming of tourists, random piling of solid wastes, and excessive development of tourism resources. Scenic spots are faced with heavy water, soil, air, and noise pollutions. These environmental pollution problems in scenic spots not only seriously threaten ecological safety but also become key constraints against the sustainable development of the tourism industry. This study analyzes four aspects of tourism-induced environmental pollutions in Shandong Province, China. Moreover, it builds a tourism economy-ecological environment coordination degree model and an index system. It then estimates the tourism economic and ecological environment coordination degree of Shandong Province from 2002 to 2019. Results demonstrate that water, soil, air, and noise pollutions are four aspects of environmental pollutions caused by the tourism industry. The D value in Shandong Province increases from 0.100 in 2002 to 0.995 in 2019 , indicating the successful transition from extreme imbalance to high-quality balance. Deepening studies on enriching the tourism industry and regional economic subsystems and subsequently exploring the temporal differences in coupling and coordination between the tourism industry and regional economy are recommended.

\section{REFERENCES}

Ahmad, F., Draz, M., Su, L., Ozturk, I. and Rauf, A. 2018. Tourism and environmental pollution: evidence from the one belt one road provinces of western China. Sustainability, 10(10).

Burger, J. 2000. Landscapes, tourism, and conservation. Science of the Total Environment, 249(1-3): 39-49.

Diakoulaki, D., Mavrotas, G. and Papayannakis, L. 1995. Determining objective weights in multiple criteria problems: The critic method. Computers \& Operations Research, 22(7): 763-770.

Greiner, A., Feichtinger, G., Haunschmied, J. L., Kort, P. M. and Hartl, R. F. 2001. Optimal periodic development of a pollution generating tourism industry. European Journal of Operational Research, 134(3): 582-591.

Huang, G., Zhou, W. and Ali, S. 2011. Spatial patterns and economic contributions of mining and tourism in biodiversity hotspots: a case study in China. Ecological Economics, 70(8): 1492-1498. 
Jang, Y. C., Hong, S., Lee, J., Lee, M. J. and Shim, W. J. 2014. Estimation of lost tourism revenue in Geoje island from the 2011 marine debris pollution event in South Korea. Marine Pollution Bulletin, 81(1): 49-54.

Kan, A. K., Li, G. Q., Yang, X., Zeng, Y. L., Tesren, L. and He, J. 2018. Ecological vulnerability analysis of Tibetan towns with tourism-based economy: a case study of the Bayi District. Journal of Mountain Science, 15(5): 1101-1114.

Kocasoy, G., Mutlu, H. and Alagöz, B. 2008. Prevention of marine environment pollution at the tourism regions by the application of a simple method for the domestic wastewater. Desalination, 226(1-3): 21-37.

Lai, Z., Ge, D., Xia, H., Yue, Y. and Wang, Z. 2020. Coupling coordination between environment, economy and tourism: a case study of china. PLOS ONE, 15 (2), e0228426.

Li, L., Yang, Q., Sun, C., Xie, X. and Zhang, F. 2021. Coupling coordinated evolution and forecast of tourism-urbanization-ecological environment: the case study of Chongqing, china. Mathematical Problems in Engineering, (3): 1-15.

Luo, Y., Wang, Z., Wang, J., Liao, Z. and Hu, M. 2017. Measuring decarbonated development of tourist attractions associated with ecological environment and tourism economy. Journal of Intelligent and Fuzzy Systems, 33(7): 1-10.
Wilson, S. P. and Verlis, K. M. 2017. The ugly face of tourism: marine debris pollution linked to visitation in the southern great barrier reef, Australia. Marine Pollution Bulletin, 117(1-2): 239.

Yang, X., Zhang, D., Liu, L., Niu, J., Zhang, X. and Wang, X. 2021. Development trajectory for the temporal and spatial evolution of the resilience of regional tourism environmental systems in 14 cities of Gansu province, china. Environmental Science and Pollution Research, 1-22.

Yuan, Y., Jin, M., Ren, J., Hu, M. and Ren, P. 2014. The dynamic coordinated development of a regional environment-tourism-economy system: a case study from western Hunan province, China. Sustainability, 6(8): 5231-5251.

Zeng, J., Wen, Y., Bi, C. and Feiock, R. 2021. Effect of tourism development on urban air pollution in china: the moderating role of tourism infrastructure. Journal of Cleaner Production, 280: 124397.

Zhang, L. and Gao, J. 2016. Exploring the effects of international tourism on china's economic growth, energy consumption and environmental pollution: evidence from a regional panel analysis. Renewable and Sustainable Energy Reviews, 53: 225-234.

Zheng, Q., Kuang, Y. and Huang, N. 2016. Coordinated development between urban tourism economy and transport in the Pearl river delta, China. Sustainability, 8(12): 1338-1338. 